\title{
Blind estimation of carrier frequency offset, I/Q imbalance and DC offset for OFDM systems
}

Tao Liu ${ }^{1 *}$ and Hanzhang Li $^{2}$

\begin{abstract}
Sensitivity to carrier frequency offset (CFO) is one of the biggest drawbacks of orthogonal frequency division multiplexing (OFDM) system. A lot of CFO estimation algorithms had been studied for compensation of CFO in OFDM system. However, with the adoption of direct-conversion architecture (DCA), which introduces additional impairments such as dc offset (DCO) and in-phase/quadrature (I/Q) imbalance in OFDM system, the established CFO estimation algorithms suffer from performance degradation. In our previous study, we developed a blind CFO, I/Q imbalance and DCO estimation algorithm for OFDM systems with DCA. In this article, we propose an alternative algorithm with reduced computation complexity and better accuracy. Performance of the proposed algorithm is demonstrated by simulations.
\end{abstract}

\section{Introduction}

As one of the most popular technologies for wireless communications, orthogonal frequency division multiplexing (OFDM) [1], on the one hand, has attractive advantages such as high spectrum efficiency, robustness to multi-path fading and efficient implementation based on fast Fourier transform, which make it suitable for wide-band wireless communications. However, on the other hand, OFDM suffers from performance degradation caused by carrier frequency offset (CFO) which damages the orthogonality among sub-carriers and introduces inter-carrier interference [2]. Since compensation of CFO is straightforward as long as estimate of CFO can be obtained, a lot of efforts have been taken on algorithms for CFO estimation in OFDM systems [3-10]. The established algorithms can be divided into two categories, data-aided and blind (or non-dataaided). The data-aided algorithms, e.g., [3], can achieve better performance than the blind ones [4-10] at costs of data rate reduction due to transmission of pilots or training sequences. What we are concerned with in this article are blind estimation algorithms that on the contrary do not depend on pilots or training sequences and therefore retain data rate of system. Among the blind algorithms, the MUSIC-like algorithm [5] was widely

\footnotetext{
* Correspondence: tgyxliu@gmail.com

${ }^{1}$ School of Automation \& Electrical Engineering, University of Science and Technology Beijing, Beijing, 100083, China

Full list of author information is available at the end of the article
}

adopted in literatures [6,11-13] as baseline due to the facts that first it was proved to be the maximum likelihood (ML) algorithm for CFO estimation in OFDM system under fading channel [7]; second it can be applied for both constant modulus modulation, e.g., phase shift keying (PSK), and inconstant modulus modulation, e.g., quadrature amplitude modulation (QAM), on sub-carriers; third it is available for estimation of both integer $\mathrm{CFO}$ and fractional CFO; and finally it achieves reasonable compromise among performance and complexity within single OFDM symbol duration.

With the evolution of wireless transceiver, direct-conversion architecture (DCA) had been widely adopted in wireless terminals especially mobile terminals to replace the superheterodyne architecture [14-16]. DCA on the one hand simplifies the design of RF front-end and reduces costs, but on the other hand introduces disturbances such as in-phase/quadrature $(\mathrm{I} / \mathrm{Q})$ imbalance and dc offset (DCO), etc., [14-17]. I/Q imbalance and DCO may not only cause interference by themselves but also violate established CFO estimation algorithms. A number of researchers had studied different approaches for joint estimation of CFO along with I/Q imbalance and/ or DCO [11-13,18-24]. Among these approaches, the blind algorithms [11-13] are of our great interests. Lin et al. [11] developed the ML-equivalent nullspace based estimator (NBE) for joint CFO and DCO estimation and proved its equivalence to $\mathrm{ML}$ estimator. In our previous study, we studied the joint estimation of CFO and I/Q 


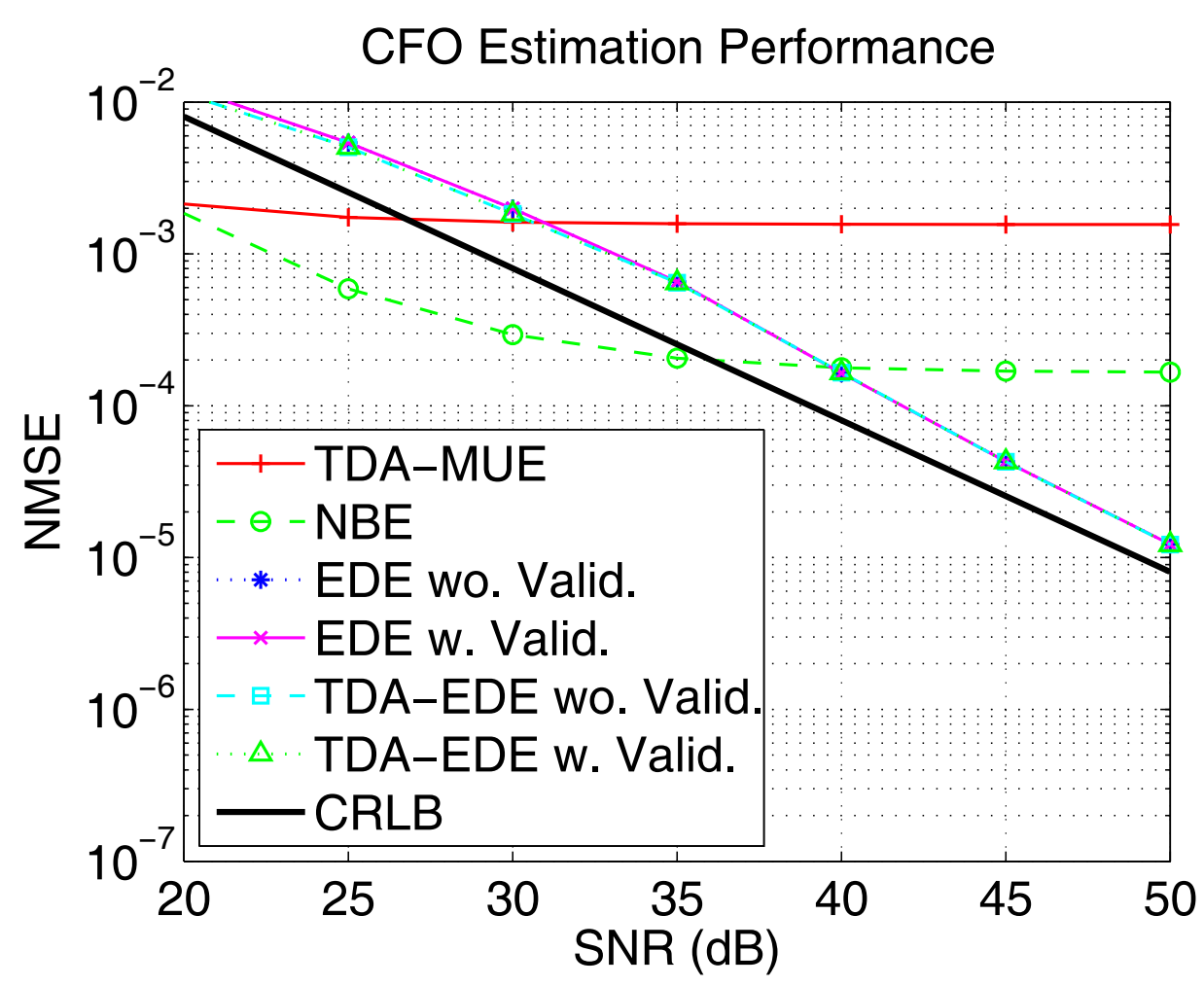

Figure 1 NMSE of CFO estimation versus SNR. CFO estimation performance with $\varepsilon=0.1,|d|^{2}=0.1,|\gamma|=0.1227$.

imbalance by exploring the similarity between the ideal OFDM signal and its mirror signal generated by I/Q imbalance [12]. Recently, we investigated the coexistence of CFO, I/Q imbalance and DCO in OFDM system and presented an eigen-decomposition based estimator (EDE) [13] for all the three parameters.

In this article, we further study joint estimation of CFO, I/Q imbalance and DCO in OFDM systems and propose an alternative blind algorithm, EDE with time domain average (TDA-EDE). When compared with EDE, TDA-EDE achieves improvements in three aspects. First, it reduces computation efforts of the estimation to around $1 / 3$ of that of EDE. Second, it improves accuracy of I/Q imbalance estimation within the whole signal to noise ratio (SNR) range of interest. Third, it significantly improves accuracy of DCO estimation with low-to-medium SNR. These improvements are achieved by compensation of DCO before estimation of CFO and I/Q imbalance, and validation of estimated I/Q imbalance according to its practical range.

The rest of this article is organized as follows. First, model of OFDM system with CFO, I/Q imbalance and DCO is established in Section 2. In Section 3, the EDE algorithm is briefly revisited and the proposed TDAEDE algorithm is then developed based on EDE and NBE in Section 4. In Section 5, simulation results and corresponding analysis are provided to demonstrate the performance of TDA-EDE. Finally, conclusions are drawn in the last section.

\section{Model of OFDM system with CFO, I/Q imbalance and DCO}

Consider an OFDM system with totally $N$ sub-carriers, among which $K$ sub-carriers occupied by data transmission are referred to as real sub-carriers, and the other $N$ - $K$ unoccupied ones are referred to as virtual sub-carriers. Let $T_{s} \triangleq \frac{T}{N}$ denotes the sampling rate in digital signal processing (DSP) stage, where $T$ is the duration of OFDM block without cyclic prefix (CP). After CP removal, the received samples that belong to the $m$ th OFDM block can be expressed as

$$
\begin{gathered}
r(n, m)=\alpha e^{j \phi(m)} \sum_{k \in C_{r}} H(k, m) S(k, m) e^{j \frac{2 \pi}{N}(k+\varepsilon) n} \\
+\beta^{*} e^{-j \phi(m)} \sum_{k \in C_{r}} H^{*}(k, m) S^{*}(k, m) e^{-j \frac{2 \pi}{N}(k+\varepsilon) n}+d+w(n, m), \\
n=0, \ldots, N-1,
\end{gathered}
$$

where $k$ refers to the index of sub-carrier, $C_{r} \triangleq\left\{k_{0}, \ldots, k_{K-1}\right\}$ is the indices set of all the $K$ real sub-carriers. $S(k, m)$ is the modulated symbol mapped onto the $k$ th sub-carrier of $m$ th OFDM block and $H(k$, 
$m)$ is the corresponding frequency domain channel response. Both $S(k, m)$ and $H(k, m)$ are assumed to be zero-mean independent random variables. $\varphi(m) \triangleq 2 \pi \varepsilon(m$ $\left.\left(N_{C P}+N\right)+N_{C P}\right) / N$ denotes a cumulative phase offset, where $\varepsilon$ and $N_{C P}$ refer to the CFO normalized to subcarrier spacing and length of $\mathrm{CP}$ in samples, respectively. The impacts of I/Q imbalance on OFDM signal are characterized by two parameters $\alpha$ and $\beta$, which were widely employed in literatures $[12,13,21]$. Since only the ratio between $\alpha$ and $\beta$ is needed for I/Q imbalance compensation, I/Q imbalance in this article is defined as $\gamma \triangleq \frac{\beta}{\alpha}$. DCO and zero-mean AWGN are denoted by the last two terms $d$ and $w(n, m)$, respectively. For notation simplicity, (1) is rewritten as

$$
\mathbf{r}_{N}(m)=\mathbf{P}_{N}(\varepsilon) \mathbf{U}_{N} \mathbf{x}_{K}(m) \alpha+\mathbf{P}_{N}(-\varepsilon) \mathbf{U}_{N}^{*} \mathbf{x}_{K}^{*}(m) \beta^{*}+1_{N} d+\mathbf{w}_{N}(m),
$$

where $\mathbf{r}_{N}(m) \triangleq[r(0, m), \ldots, r(N-1, m)]^{T}, \mathbf{x}_{K}(m) \triangleq[H$ $\left.\left(k_{0}, m\right) S\left(k_{0}, m\right), \ldots, H\left(k_{K-1}, m\right) S\left(k_{K-1}, m\right)\right]^{T} e^{j \varphi(m)}$ and $\mathbf{w}_{N}$ $(m) \triangleq[w(0, m), \ldots, w(N-1, m)]^{T} . \mathbf{l}_{N}$ denotes an all ones column vector of length $N$. The inverse discrete Fourier transform (IDFT) on real sub-carriers are denoted by an $N \times K$ matrix $\mathbf{U}_{N}$, whose $(n, l)$ th entry is $e^{j \frac{2 \pi}{N}} u_{l} n$ with $u_{l} \in C_{r}$. The diagonal matrix $\mathbf{P}_{N}(\varepsilon) \triangleq \operatorname{diag}\left\{1, e^{j \frac{2 \pi}{N} \varepsilon}, \ldots, e^{j \frac{2 \pi(N-1)}{N} \varepsilon}\right\}$ represents the phase offset caused by CFO.

\section{Eigen-decomposition based estimator (EDE)}

In our recent study [13], we proposed an eigen-decomposition based blind estimator (EDE) for joint estimation of CFO, I/Q imbalance and DCO in OFDM systems. The basic idea of EDE can be summarized as (1) the impacts of I/Q imbalance and DCO on OFDM signal can be compensated by linear combination of the received signal, its complex conjugate, and an arbitrary dc signal; (2) the established MUSIC-like CFO estimation algorithm [5] can then be applied to compensated signal. Based on the ideas, a key cost function was first constructed in EDE as

$$
f_{\mathrm{EDE}}\left(v, \mathbf{a}_{3}\right)=\sum_{m}\left\|\mathbf{V}_{N}^{H} \mathbf{P}_{N}(-v) \mathbf{R}_{3}(m) \mathbf{a}_{3}\right\|^{2},
$$

where $\mathbf{V}_{N}$ is an $N \times(N-K)$ matrix, whose $(n, l)$ th entry

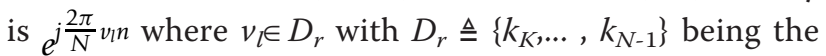
indices set of all the $N-K$ virtual sub-carriers. Note that the columns of $\mathbf{U}_{N}$ are orthogonal to those of $\mathbf{V}_{N}$, i.e., $\mathbf{V}_{N}^{H} \mathbf{U}_{N}=\mathbf{0}_{N} \mathbf{R}_{3}(m) \triangleq\left[\mathbf{r}_{N}(m), \mathbf{r}_{N}^{*}(m), \mathbf{l}_{N}\right]$ consists of the received signal, its complex conjugate, and a unit dc signal for the linear combination with $\mathbf{a}_{3} \triangleq[a(0), a(1), a$ $(2)]^{T}$ being the corresponding weighting factors. The cost function in (3) is actually the same as that proposed in [5] except that the received signal $\mathbf{r}_{N}(m)$ is replaced by the combination $\mathbf{R}_{3}(m) \mathbf{a}_{3}$. It had been illustrated in [13] that $f_{\mathrm{EDE}}\left(v, \mathbf{a}_{3}\right)$ achieves its minimum when $v= \pm \varepsilon$ and $\mathbf{a}_{3}=\mathbf{g}_{3}$ with $\mathbf{g}_{3}$ being the optimal weighting vector that can completely cancel the I/Q imbalance and DCO in received signal [13]. Therefore, CFO estimation can be achieved by minimization of $f_{\mathrm{EDE}}\left(v, \mathbf{a}_{3}\right)$ as

$$
\hat{\varepsilon}_{a, E D E}=\underset{v}{\arg \min } \lambda_{\min }\left\{\boldsymbol{\Omega}_{3}(v)\right\},
$$

$$
\text { where } \boldsymbol{\Omega}_{3}(v) \triangleq \sum_{m} \mathbf{R}_{3}^{H}(m) \mathbf{P}_{N}(v) \mathbf{V}_{N} \mathbf{V}_{N}^{H} \mathbf{P}_{N}(-v) \mathbf{R}_{3}(m) .
$$

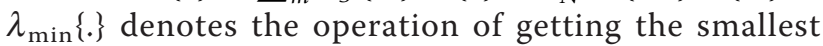
eigenvalue of matrix. To get rid of the sign ambiguity in $\hat{\varepsilon}_{a, \mathrm{EDE}}$, a metric $T_{\mathrm{CFO}}$, EDE was employed in EDE [13]. Finally, the CFO estimate without sign ambiguity was obtained by

$$
\hat{\varepsilon}_{\mathrm{EDE}}=-\operatorname{sign}\left(T_{\mathrm{CFO}, \mathrm{EDE}}\right) \hat{\varepsilon}_{a, \mathrm{EDE}},
$$

The eigenvector corresponding to the smallest eigenvalue of $\boldsymbol{\Omega}_{3}\left(\hat{\varepsilon}_{\mathrm{EDE}}\right)$ is taken as estimate of $\mathbf{g}_{3}$, from which $\mathrm{I} / \mathrm{Q}$ imbalance and DCO can be derived [13].

\section{EDE with time domain average (TDA-EDE)}

When compared with EDE, TDA-EDE achieves improvements in three aspects. First, it reduces computation efforts of the estimation to around $1 / 3$ of that of EDE. Second, it improves the accuracy of I/Q imbalance estimation within the whole SNR range of interest. Third, it significantly improves the accuracy of DCO estimation with low-to-medium SNR.

\subsection{Coarse estimation and compensation of DCO by time domain average}

The first improvement of TDA-EDE comes from compensation of DCO before estimation of CFO and I/Q imbalance. Inspired by [11], we first make a coarse estimation for DCO by time domain average as

$$
\begin{aligned}
\hat{d}(m) & =\frac{1}{N} \sum_{n=0}^{N-1} r(n, m) \\
& =\frac{1}{N} \mathbf{1}_{N}^{T} \mathbf{r}_{N}(m),
\end{aligned}
$$

Then we cancel it from the received signal according to

$$
\mathbf{y}_{N}(m) \triangleq \mathbf{r}_{N}(m)-\mathbf{1}_{N} \hat{d}(m) .
$$

By substituting (6) into (7), we have

$$
\begin{aligned}
\mathbf{y}_{N}(m) & =\mathbf{r}_{N}(m)-\frac{1}{N} \mathbf{1}_{N} \mathbf{1}_{N}^{T} \mathbf{r}_{N}(m) \\
& =\mathbf{E}_{N} \mathbf{r}_{N}(m),
\end{aligned}
$$


where $\mathbf{E}_{N} \triangleq \mathbf{I}_{N}-\frac{1}{N} \mathbf{1}_{N} \mathbf{1}_{N}^{T}$ with $\mathbf{I}_{N}$ being the $N \times N$ identity matrix. Due to the fact that $\mathbf{E}_{N} \mathbf{l}_{N}=\mathbf{0}$, substituting (2) into (8) yields

$$
\mathbf{y}_{N}(m)=\mathbf{E}_{N} \mathbf{P}_{N}(\varepsilon) \mathbf{U}_{N} \mathbf{x}(m) \alpha+\mathbf{E}_{N} \mathbf{P}_{N}(-\varepsilon) U_{N}^{*} \mathbf{x}^{*}(m) \beta^{*}+\mathbf{E}_{N} \mathbf{w}_{N}(m) .
$$

\subsection{Estimation of CFO by eigen-decomposition}

We can find in (9) that not only DCO is completely removed but also a key property of (2) that the desired signal component with $\mathrm{CFO} \varepsilon$, i.e., the first term on the right-hand side (RHS), and its mirror component with CFO $-\varepsilon$ introduced by I/Q imbalance, i.e., the second term on the RHS, are conjugates to each other up to a scaling factor is remained. As analyzed in [13], either of the two signal components can be removed by linear combination

$$
\mathbf{b}_{N}(m) \triangleq \mathbf{Y}_{2}(m) \mathbf{g}_{2}
$$

where

$$
\mathbf{Y}_{2}(m) \triangleq\left[\mathbf{y}_{N}(m), \mathbf{y}_{N}^{*}(m)\right] .
$$

The optimal weighting vector denoted by $\mathbf{g}_{2} \triangleq[g(0), g$ $(1)]^{T}$ should satisfy either

$$
g(0) \beta^{*}+g(1) \alpha^{*}=0
$$

to mitigate the component with $\mathrm{CFO} \varepsilon$, or

$$
g(0) \alpha+g(1) \beta=0,
$$

to suppress the component with $\mathrm{CFO}-\varepsilon$. In absence of I/Q imbalance, we can directly apply NBE to the DCO compensated signal $\mathbf{y}_{N}(m)$. The cost function should be constructed as [11]

$$
\begin{aligned}
f_{N B E}(v) & =\sum_{m}\left\|\tilde{\mathbf{V}}_{N}^{H}(v) \mathbf{y}_{N}(m)\right\|^{2} \\
& =\sum\left\|\tilde{\mathbf{V}}_{N}^{H}(v) \mathbf{E}_{N} \mathbf{r}_{N}(m)\right\|^{2},
\end{aligned}
$$

where the columns of $\tilde{\mathbf{V}}_{N}(v)$ are eigenvectors corresponding to the zero eigenvalues of $\mathbf{Q}_{N}(v) \triangleq \mathbf{E}_{N} \mathbf{P}_{N}(v) \mathbf{U}_{N} \mathbf{U}_{N}^{H} \mathbf{P}_{N}(-v) \mathbf{E}_{N}$. As $\mathbf{E}_{N}$ is of rank $N$ $1, \mathbf{Q}_{N}(v)$ has at least one zero eigenvalue. In order to involve the compensation of $\mathrm{I} / \mathrm{Q}$ imbalance, a new cost function is constructed for TDA-EDE as

$$
f_{\mathrm{TDA}-\mathrm{EDE}}\left(v, \mathbf{a}_{2}\right)=\sum_{m}\left\|\tilde{\mathbf{V}}_{N}^{H}(v) \mathbf{Y}_{2}(m) \mathbf{a}_{2}\right\|^{2},
$$

where $\mathbf{a}_{2} \triangleq[a(0), a(1)]^{T}$. By substituting (8) into (11), we have

$$
\mathbf{Y}_{2}(m)=\mathbf{E}_{N} \mathbf{R}_{2}(m),
$$

where $R_{2}(m) \triangleq\left[\mathbf{r}_{N}(m), \mathbf{r}_{N}^{*}(m)\right]$. Consequently, (15) can be rewritten as

$$
f_{\mathrm{TDA}-E D E}\left(v, \mathbf{a}_{2}\right)=\sum_{m}\left\|\tilde{\mathbf{V}}_{N}^{H}(v) \mathbf{E}_{N} \mathbf{R}_{2}(m) \mathbf{a}_{2}\right\|^{2} .
$$

Based on the constructed cost function, estimation of $\mathrm{CFO}$ and optimal weighting factor can be achieved by the following optimization approach as

$$
\begin{aligned}
\left(\hat{\varepsilon}, \hat{\mathbf{g}}_{2}\right) & =\underset{\left(v, \mathbf{a}_{2}\right)}{\arg \min } f_{\mathrm{TDA}-E D E}\left(v, \mathbf{a}_{2}\right) \\
& =\underset{\left(v, \mathbf{a}_{2}\right)}{\arg \min } \sum_{m}\left\|\tilde{\mathbf{V}}_{N}^{H}(v) \mathbf{E}_{N} \mathbf{R}_{2}(m) \mathbf{a}_{2}\right\|^{2} \\
& =\underset{\left(v, \mathbf{a}_{2}\right)}{\arg \min }\left\{\mathbf{a}_{2}^{H} \boldsymbol{\Omega}_{2}(v) \mathbf{a}_{2}\right\},
\end{aligned}
$$

where $\boldsymbol{\Omega}_{2}(v) \triangleq \sum_{m} \mathbf{R}_{2}^{H}(m) \mathbf{E}_{N} \tilde{\mathbf{V}}_{N}(v) \tilde{\mathbf{V}}_{N}^{H}(v) \mathbf{E}_{N} \mathbf{R}_{2}(m)$. To avoid homogenous solution $\hat{\mathbf{g}}_{2}=0_{2}$, we impose a constraint $\left\|\mathbf{a}_{2}\right\|^{2}=1$ to (17). The well-known solution to this constrained minimization is [25]

$$
\hat{\varepsilon}_{a, T D A-E D E}=\underset{v}{\arg \min } \lambda_{\min }\left\{\boldsymbol{\Omega}_{2}(v)\right\},
$$

and $\hat{\mathbf{g}}_{2}$ equals to the eigenvector corresponding to the smallest eigenvalue of $\boldsymbol{\Omega}_{2}\left(\hat{\varepsilon}_{a, \mathrm{TDA}-\mathrm{EDE}}\right)$. In case that $\boldsymbol{\Omega}_{2}\left(\hat{\varepsilon}_{a, \mathrm{TDA}-\mathrm{EDE}}\right)$. has multiple minimum eigenvalues, $\hat{\mathbf{g}}_{2}$ can be randomly selected from the eigenvectors corresponding to the minimum eigen-value. Actually, since $\boldsymbol{\Omega}_{2}\left(\hat{\varepsilon}_{a, \mathrm{TDA}-\mathrm{EDE}}\right)$. constitutes random data, it is of little possibility to have multiple minimum eigenvalues. Consequently, $\hat{\mathbf{g}}_{2}$ obtained under such case has little influence of average performance.

\subsection{Sign ambiguity of CFO estimation and its solution}

It should be noted that the CFO estimate $\hat{\varepsilon}_{a, \mathrm{TDA}-\mathrm{EDE}}$ obtained by (19) will involve sign ambiguity, i.e., $\hat{\varepsilon}_{a, \mathrm{TDA}-\mathrm{EDE}}$ may approaches either $\varepsilon$ or $-\varepsilon$. As mentioned in Section 3, similar problem was also encountered by EDE [13]. From physical point of view, the ambiguity comes from the fact that the original received signal (2) contains component with $\mathrm{CFO} \varepsilon$ as well as component with CFO $-\varepsilon$. From mathematical point of view, it can be deduced that $\hat{\mathbf{V}}_{N}^{H}(\varepsilon) \mathbf{E}_{N} \mathbf{P}_{N}(\varepsilon) \mathbf{U}_{N}=\hat{\mathbf{V}}_{N}^{H}(-\varepsilon) \mathbf{E}_{N} \mathbf{P}_{N}(-\varepsilon) \mathbf{U}_{N}^{*}=\mathbf{0}_{N}$. Consequently, both $\boldsymbol{\Omega}_{2}(\varepsilon)$ and $\boldsymbol{\Omega}_{2}(-\varepsilon)$ are rank deficient and therefore have eigenvalues of zero in absence of noise. To distinguish from sign ambiguity in CFO estimation, we take a similar approach as EDE with however a different metric $T_{\text {CFO,TDA- EDE }} \triangleq \sum_{m}\left\{\| \tilde{\mathbf{V}}_{N}^{H}\left(\hat{\varepsilon}_{a, T D A}-\right.\right.$ EDE $\left.) \mathbf{y}_{N}(m)\left\|^{2}-\right\| \tilde{\mathbf{V}}_{N}^{H}\left(-\hat{\varepsilon}_{a, T D A-E D E}\right) \mathbf{y}_{N}(m) \|^{2}\right\}$. Substituting (9) into $T_{\text {CFO,TDA-EDE }}$ yields that 


$$
\begin{aligned}
T_{\mathrm{CFO}, \mathrm{TDA}-\mathrm{EDE}}= & \sum_{m}\left\{\| \tilde{\mathbf{V}}_{N}^{H}\left(\hat{\varepsilon}_{a, \mathrm{TDA}-\mathrm{EDE}}\right) \mathbf{E}_{N} \mathbf{P}_{N}(\varepsilon) U_{N} \mathbf{x}(m) \alpha\right. \\
& +\tilde{\mathbf{V}}_{N}^{H}\left(\hat{\varepsilon}_{a, \mathrm{TDA}-\mathrm{EDE}}\right) \mathbf{E}_{N} \mathbf{P}_{N}(-\varepsilon) \mathbf{U}_{N}^{*} \mathbf{x}^{*}(m) \beta^{*} \\
& +\tilde{\mathbf{V}}_{N}^{H}\left(\hat{\varepsilon}_{a, \mathrm{TDA}-\mathrm{EDE}}\right) \mathbf{E}_{N} \mathbf{w}_{N}(m) \|^{2} \\
& -\| \tilde{\mathbf{V}}_{N}^{H}\left(-\hat{\varepsilon}_{a, \mathrm{TDA}-\mathrm{EDE}}\right) \mathbf{E}_{N} \mathbf{P}_{N}(\varepsilon) U_{N} \mathbf{x}(m) \alpha \\
& +\tilde{\mathbf{V}}_{N}^{H}\left(-\hat{\varepsilon}_{a, \mathrm{TDA}-\mathrm{EDE}}\right) \mathbf{E}_{N} \mathbf{P}_{N}(-\varepsilon) U_{N}^{*} \mathbf{x}^{*}(m) \beta^{*} \\
& \left.+\tilde{\mathbf{V}}_{N}^{H}\left(-\hat{\varepsilon}_{a, \mathrm{TDA}-\mathrm{EDE}}\right) \mathbf{E}_{N} \mathbf{w}_{N}(m) \|^{2}\right\}
\end{aligned}
$$

Recall that $\tilde{\mathbf{V}}_{N}(v)$ consists of eigenvectors corresponding to the zero eigenvalues of $\mathbf{Q}_{N}(v)$, we have $\tilde{\mathbf{V}}_{N}^{H}(v) \mathbf{E}_{N} \mathbf{P}_{N}(v) \mathbf{U}_{N}=\mathbf{0}_{N}$. In absence of noise, (20) can be simplified to

$$
T_{\mathrm{CFO}, \mathrm{TDA}-\mathrm{EDE}}\left(\hat{\varepsilon}_{a, \mathrm{TDA}-\mathrm{EDE}}=\varepsilon\right)=p|\beta|^{2}-q|\alpha|^{2},
$$

or

$$
T_{\mathrm{CFO}, \mathrm{TDA}-\mathrm{EDE}}\left(\hat{\varepsilon}_{a, \mathrm{TDA}-\mathrm{EDE}}=-\varepsilon\right)=q|\alpha|^{2}-p|\beta|^{2},
$$

where $p \triangleq \sum_{m}\left\|\tilde{\mathbf{V}}_{N}^{H}(\varepsilon) \mathbf{E}_{N} \mathbf{P}_{N}(-\varepsilon) \mathbf{U}_{N}^{*} \mathbf{x}^{*}(m)\right\|^{2}=\sum_{m}\left\|\tilde{\mathbf{V}}_{N}^{T}(\varepsilon) \mathbf{E}_{N} \mathbf{P}_{N}(\varepsilon) \mathbf{U}_{N} \mathbf{x}(m)\right\|^{2}$ and $q \triangleq \sum_{m}\left\|\tilde{\mathbf{V}}_{N}^{H}(-\varepsilon) \mathbf{E}_{N} \mathbf{P}_{N}(\varepsilon) \mathbf{U}_{N} \mathbf{x}(m)\right\|^{2}$. For most practical cases, $p$ and $q$ are comparable. If the real subcarriers are located symmetrically about dc, or mathematically if any column of $\mathbf{U}_{N}$ is also a column of $\mathbf{U}_{N}^{*}$, we have $\boldsymbol{\Omega}_{2}^{*}(\varepsilon)=\mathbf{E}_{N} \mathbf{P}_{N}(-\varepsilon) \mathbf{U}_{N}^{*} \mathbf{U}_{N}^{T} \mathbf{P}_{N}(\varepsilon) \mathbf{E}_{N}=\mathbf{E}_{N} \mathbf{P}_{N}(-\varepsilon) \mathbf{U}_{N} \mathbf{U}_{N}^{H} \mathbf{P}_{N}(\varepsilon) \mathbf{E}_{N}=\boldsymbol{\Omega}_{2}(-\varepsilon)$ Recall that $\tilde{\mathbf{V}}_{N}^{T}(\varepsilon)$ and $\tilde{\mathbf{V}}_{N}^{H}(-\varepsilon)$ consist of the eigenvectors of $\boldsymbol{\Omega}_{2}^{*}(\varepsilon)$ and $\boldsymbol{\Omega}_{2}(-\varepsilon)$, respectively, we have $\tilde{\mathbf{V}}_{N}^{*}(\varepsilon) \tilde{\mathbf{V}}_{N}^{T}(\varepsilon)=\tilde{\mathbf{V}}_{N}(-\varepsilon) \tilde{\mathbf{V}}_{N}^{H}(-\varepsilon)$ which leads to $p=q$. And because $|\alpha|^{2} \gg|\beta|^{2}[14-16]$ in practice, the sign ambiguity can be distinguished by

$$
\begin{aligned}
T_{C F O, T D A-E D E} & \hat{\varepsilon}_{a, T D A-E D E}=\varepsilon \\
\hat{\varepsilon}_{a, T D A-E D E} & =-\varepsilon
\end{aligned}
$$

The final CFO estimate is obtained as

$$
\hat{\varepsilon}_{\mathrm{TDA}-\mathrm{EDE}}=-\operatorname{sign}\left(T_{\mathrm{CFO}, \mathrm{TDA}-\mathrm{EDE}}\right) \hat{\varepsilon}_{a, \mathrm{TDA}-\mathrm{EDE}} .
$$

\subsection{Estimation and validation of $\mathrm{I} / \mathrm{Q}$ imbalance}

Integrated with the CFO estimation, the eigenvector corresponding to the smallest eigenvalue of $\boldsymbol{\Omega}_{2}\left(\hat{\varepsilon}_{a, \mathrm{TDA}-\mathrm{EDE}}\right)$. is taken as $\hat{\mathbf{g}}_{2}$, which is the estimate of the optimal weighting vector for the linear combination. Consequently, I/Q imbalance can be derived either from (12) as

$$
\hat{\gamma}_{\mathrm{TDA}-\mathrm{EDE}}=-\frac{\hat{g}^{*}(1)}{\hat{g}^{*}(0)},
$$

for the cases of $T_{\mathrm{CFO}}$,TDA-EDE $<0$, or otherwise from (13) as

$$
\hat{\gamma}_{\mathrm{TDA}-\mathrm{EDE}}=-\frac{\hat{g}(0)}{\hat{g}(1)} .
$$

The second and third improvements of TDA-EDE are achieved mainly by validation of estimated I/Q imbalance according to its practical range. Recall the fact that $|\alpha|^{2} \gg|\beta|^{2}$ [14-16] in practice, which equivalently gives the range that $|\gamma|^{2} \ll 1$. A too large $\hat{\gamma}_{T D A-E D E}$ usually indicates failure of $\mathrm{I} / \mathrm{Q}$ imbalance estimation. In TDAEDE, we validate the estimated I/Q imbalance according to a controllable threshold $T_{\text {IQI,TDA-EDE }} \in(0,1)$. If the estimated result exceeds this threshold, i.e., $\hat{\gamma}_{T D A-E D E}>T_{\mathrm{IQI}, \mathrm{TDA}-\mathrm{EDE}}$, it will be reset to $\hat{\gamma}_{T D A-E D E}=0$, which means we would prefer to ignore I/Q imbalance for such cases rather than compensate it based on the unreasonable estimation results.

\subsection{Compensation of CFO and I/Q imbalance}

Given the estimates of CFO and I/Q imbalance, their impacts can be compensated in the original received signal by

$$
\mathbf{z}_{N}(m) \triangleq \mathbf{r}_{N}(m)-\mathbf{r}_{N}^{*}(m) \hat{\gamma}_{\mathrm{TDA}-\mathrm{EDE}}^{*} .
$$

Suppose that $\hat{\gamma}_{\mathrm{TDA}-\mathrm{EDE}}=\gamma$ and then substitute (2) into (27), we have

$$
\mathbf{z}_{N}(m)=\mathbf{P}_{N}(\varepsilon) \mathbf{U}_{N} x_{K}(m)\left(\alpha-\beta \hat{\gamma}_{\mathrm{TDA}-\mathrm{EDE}}^{*}\right)+\mathbf{l}_{N} c+\tilde{\mathbf{w}}_{N}(m),
$$

where

$$
c \triangleq d-d^{*} \hat{\gamma}_{\mathrm{TDA}-E D E^{\prime}}^{*}
$$

and $\tilde{w}_{N}(m) \triangleq w_{N}(m)-w_{N}^{*}(m) \hat{\gamma}_{\mathrm{TDA}-\mathrm{EDE}}^{*}$. It can be observed from the RHS of (28) that only the desired signal component with $\mathrm{CFO} \varepsilon$ is retained.

\subsection{Fine estimation of DCO}

Suppose that $\hat{\varepsilon}_{T D A-E D E}=\varepsilon$, it can be derived from (28) that

$$
\begin{gathered}
\mathbf{V}_{N}^{H} \mathbf{P}_{N}\left(-\hat{\varepsilon}_{\mathrm{TDA}-\mathrm{EDE}}\right) z_{N}(m)=\mathbf{V}_{N}^{H} \mathbf{P}_{N}\left(-\hat{\varepsilon}_{\mathrm{TDA}-\mathrm{EDE}}\right) \mathbf{l}_{N} c \\
+\mathbf{V}_{N}^{H} \mathbf{P}_{N}\left(-\hat{\varepsilon}_{\mathrm{TDA}-\mathrm{EDE}}\right) \tilde{\mathbf{w}}_{N}(m) .
\end{gathered}
$$

Through least square (LS) approach, estimate of $c$ can be obtained by

$$
\begin{aligned}
\hat{c} & =\left[\mathbf{V}_{N}^{H} \mathbf{P}_{N}\left(-\hat{\varepsilon}_{\mathrm{TDA}-\mathrm{EDE}}\right) \mathbf{l}_{N}\right]^{+} \mathbf{V}_{N}^{H} \mathbf{P}_{N}\left(-\hat{\varepsilon}_{\mathrm{TDA}-\mathrm{EDE}}\right) \mathbf{z}_{N}(m) \\
& =\frac{\mathbf{l}_{N}^{T} \mathbf{G}_{N}\left(\hat{\varepsilon}_{\mathrm{TDA}-\mathrm{EDE}}\right) \mathbf{z}_{N}(m)}{\mathbf{l}_{N}^{T} \mathbf{G}_{N}\left(\hat{\varepsilon}_{\mathrm{TDA}-\mathrm{EDE}}\right) \mathbf{l}_{N}}
\end{aligned}
$$


where [.] $]^{+}$denotes pseudo-inversion and

$$
\mathbf{G}_{N}\left(\hat{\varepsilon}_{\mathrm{TDA}-\mathrm{EDE}}\right) \triangleq \mathbf{P}_{N}\left(\hat{\varepsilon}_{\mathrm{TDA}-\mathrm{EDE}}\right) \mathbf{V}_{N} \mathbf{V}_{N}^{H} \mathbf{P}_{N}\left(-\hat{\varepsilon}_{\mathrm{TDA}-\mathrm{EDE}}\right) .
$$

Finally, fine estimate of DCO can be derived from (29) as

$$
\hat{d}=\frac{\hat{c}+\hat{c}^{*} \hat{\gamma}_{\mathrm{TDA}-\mathrm{EDE}}^{*}}{1-\left|\hat{\gamma}_{\mathrm{TDA}-\mathrm{EDE}}\right|^{2}} .
$$

\subsection{Summary and discussion}

The steps for joint estimation of CFO, DCO, and I/Q imbalance by TDA-EDE can be summarized as:

- After CP removal, group received samples that belong to the same OFDM block to construct the vector $\mathbf{r}_{N}(m) \triangleq[r(0, m), \ldots, r(N-1, m)]^{T}$;

- Cancel the DCO in $\mathbf{r}_{N}(m)$ by time domain average according to (7) to obtain $\mathbf{y}_{N}(m)$;

- Search for the trail $v$ that minimizes the smallest eigenvalue of $\boldsymbol{\Omega}_{2}(v)$ according to (19);

- Set $\hat{\varepsilon}_{a, \mathrm{TDA}-\mathrm{EDE}}$ to the $v$ found during the search and record the eigenvector corresponding to the smallest eigenvalue of $\Omega_{2}\left(\hat{\varepsilon}_{a, \mathrm{TDA}-\mathrm{EDE}}\right)$ in $\mathbf{g}_{2}$;
- Calculate $T_{\mathrm{CFO} \text {,TDA-EDE }}$ according to its definition, and detect sign ambiguity with (23) and correct it with (24);

- Get estimate of I/Q imbalance according to (25) or (26) depending on the sign of $T_{\text {CFO,TDA-EDE }}$ and then reset it to 0 if it exceeds $T_{\text {IQI,TDA-EDE }}$ in the validation;

- Compensate CFO and I/Q imbalance according to (27) to obtain $\mathbf{z}_{N}(m)$;

- Get fine estimation of DCO according to (31) and (33).

Computation efforts of TDA-EDE are mainly determined by the eigen-decomposition of $\boldsymbol{\Omega}_{2}(v)$ in the 1-D search operation for CFO estimation, as it will execute in each searching step while other operations execute only once. Although computation of $\tilde{\mathbf{V}}_{N}^{H}(v)$ in TDA-EDE is much more time-consuming than that of $\mathbf{V}_{N}^{H} \mathbf{P}_{N}(-v)$ in EDE, both of them can be calculated and stored in advance [11] and is therefore not necessary to be computed on the run. What has to be done online for TDAEDE is the eigen-decomposition of $\boldsymbol{\Omega}_{2}(v)$. If the wellknown Power Method [25] is employed, eigen-

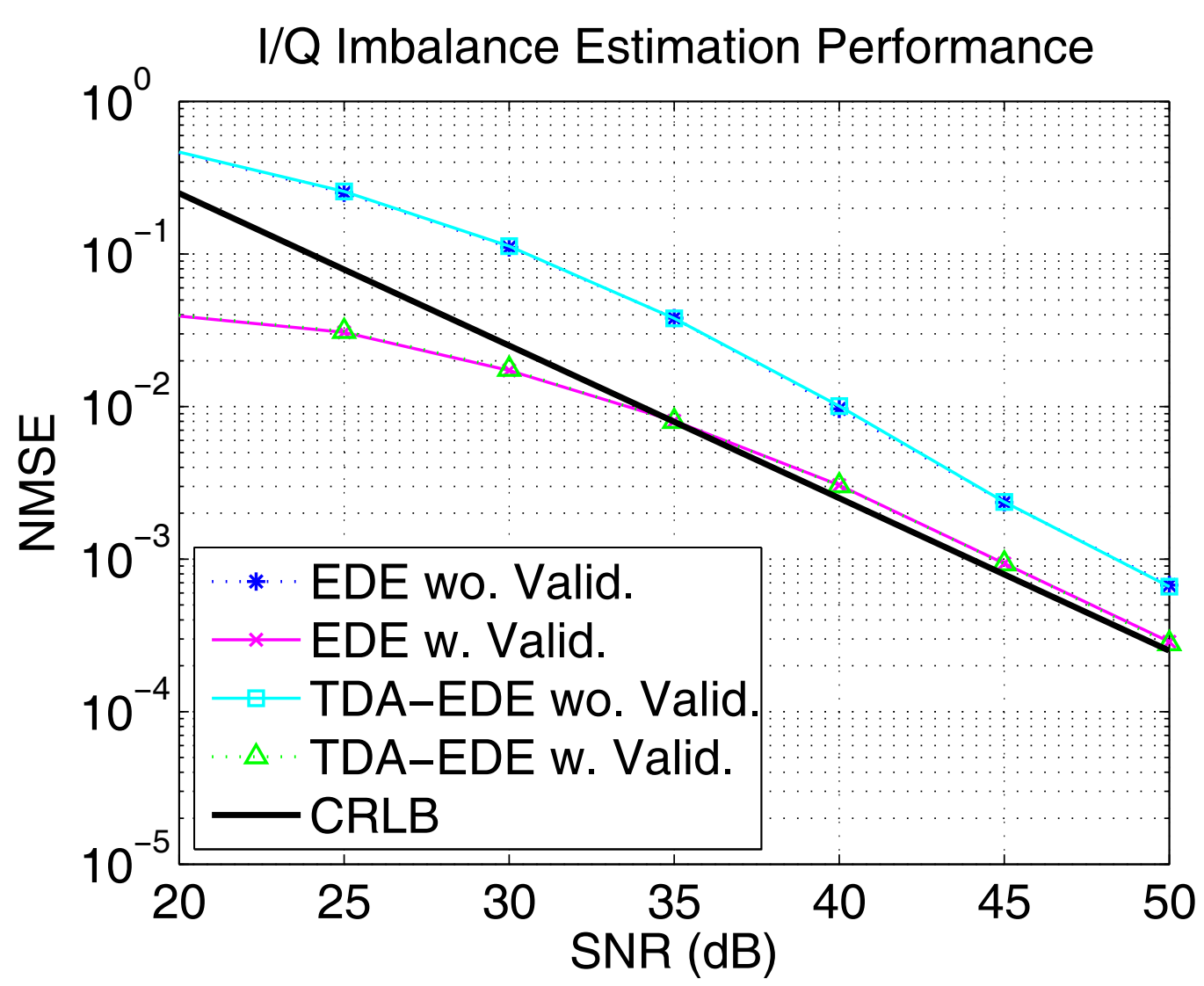

Figure 2 NMSE of I/Q imbalance estimation versus SNR. I/Q imbalance estimation performance with $\varepsilon=0.1,|d|^{2}=0.1,|\gamma|=0.1227$. 


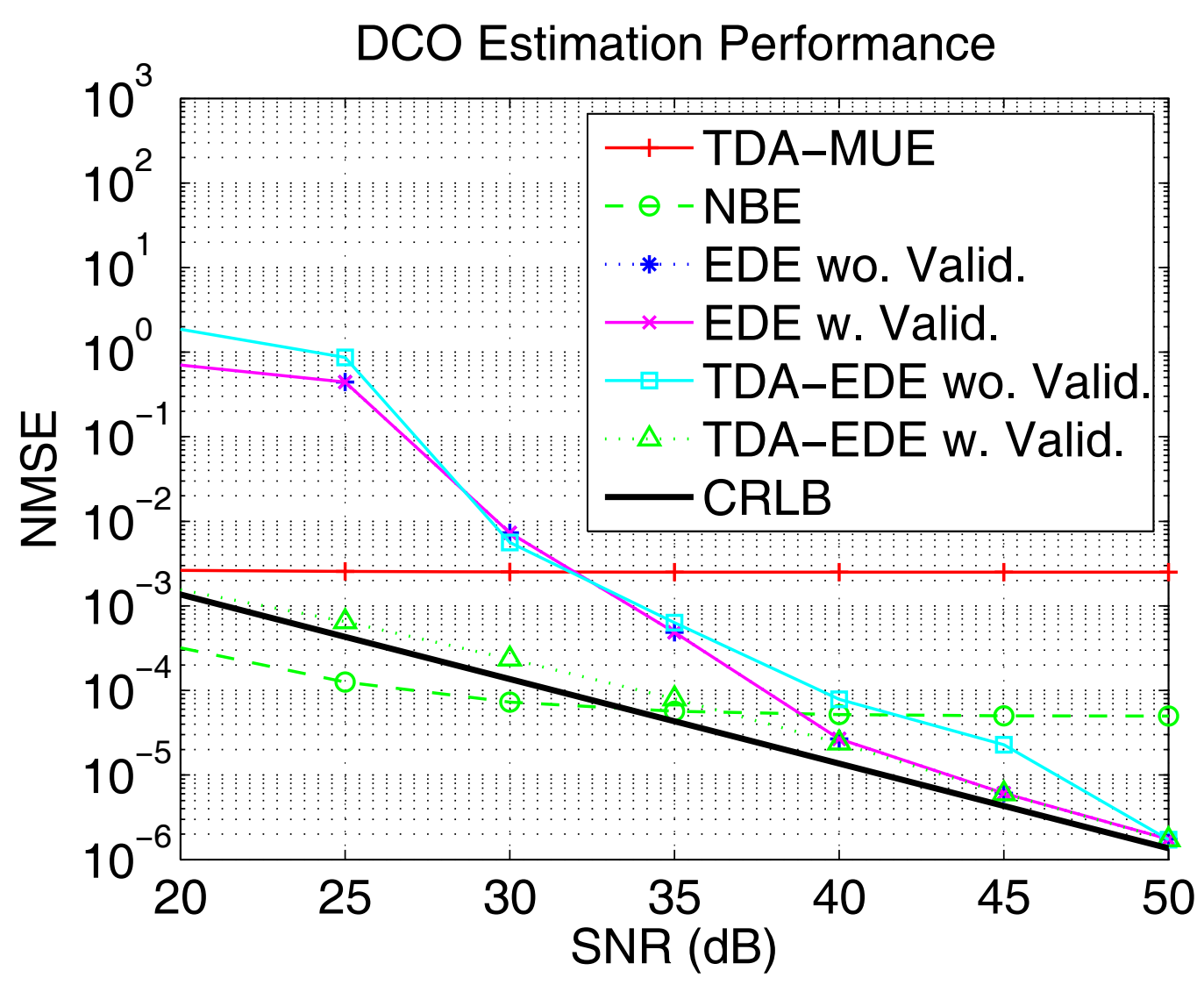

Figure 3 NMSE of DCO estimation versus SNR. DCO estimation performance with $\varepsilon=0.1,|d|^{2}=0.1,|\gamma|=0.1227$

decomposition of the $2 \times 2$ matrix $\boldsymbol{\Omega}_{2}(v)$ is of complexity $O\left(2^{3}\right)[26,27]$, while eigen-decomposition of the $3 \times$ 3 matrix $\boldsymbol{\Omega}_{3}(v)$ in EDE is of complexity $O\left(3^{3}\right)$ [26,27]. Therefore, TDA-EDE reduces about $1 / 3$ of the computation efforts compared with EDE.

\section{Simulation results}

As has been illustrated, TDA-EDE originates from NBE and EDE, which in turn come from MUE [5]. In order to demonstrate the performance of TDA-EDE, it is compared by simulations with NBE [11], EDE [13] and TDA-MUE, which compensates DCO with time domain average approach before estimation of CFO with MUE. Moreover, to illustrate the benefits of I/Q imbalance validation, EDE and TDA-EDE with and without validation are evaluated. The threshold for the validation is set to 0.5 . The OFDM system in the simulations is a WLAN [28] system with $N=64, K=$ 48, 64-QAM modulation. The frequency selective Rayleigh fading channel is set to have power delay profile $e^{-p / 5}, p=0, \ldots, 9$. The estimation performance is evaluated by normalized mean square error (NMSE) defined as $E\left\{|\hat{\varepsilon}-\varepsilon|^{2}\right\}, E\left\{|\hat{\gamma}-\gamma|^{2}\right\}$, and $E\left\{|\hat{d}-d|^{2}\right\}$, where $E\{$.$\} denotes expectation operation. To track the$ variation of CFO, I/Q imbalance and DCO as quickly as possible, only one OFDM symbol is used in the estimation. In addition to the aforementioned estimators, we also provide the corresponding Cramér-Rao lower bound (CRLB), whose derivation can be found in [13].

Performance of CFO, I/Q imbalance and DCO estimation with different estimators are shown in Figures 1, 2, and 3 , respectively. It can be observed that TDA-MUE and NBE exhibit error floor for all the estimations due to the impacts of I/Q imbalance. On the contrary, performance of both EDE and TDA-EDE achieve CRLB approximately with increasing SNR. It is also demonstrated by Figures 1, 2, and 3 that, given the reduction of computation efforts, TDA-EDE achieves the same accuracy as EDE for estimation of CFO and I/Q imbalance and outperforms EDE for DCO estimation especially with low-to-medium SNR. This is because TDAEDE refines DCO estimation with validated estimates of I/Q imbalance. Comparison between the cases with and without validation also reveals the importance of I/Q imbalance validation. 


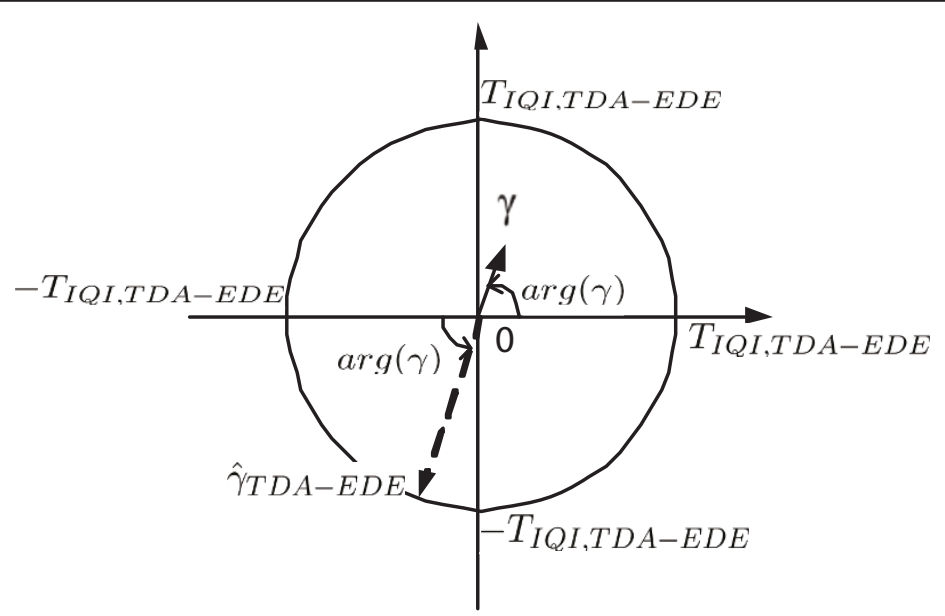

Figure 4 Illustration of the worst case for I/Q imbalance estimation.

Please note that TDA-EDE and EDE achieve better performance than CRLB for I/Q imbalance estimation with relative low SNR when validation according to practical range of $\mathrm{I} / \mathrm{Q}$ imbalance is performed. This is because that the validation makes additional error reduction. Recall that the validation will reset $\hat{\gamma}_{T D A-E D E}$ to 0 if it exceeds $T_{\text {IQI,TDA-EDE }}$, which results in NMSE of $|\gamma|^{2}$. If on the contrary $\hat{\gamma}_{T D A-E D E}$ does not exceeds $T_{\text {IQI,TDA-EDE, }}$ the worst case as shown in Figure 4 will be $\hat{\gamma}_{T D A-E D E}=T_{I Q I, T D A-E D E} e^{j[\arg (\gamma)+\pi]}$, which results in NMSE of $\left(|\gamma|+T_{\text {IQI,TDA-EDE }}\right)^{2}$. As $\left(|\gamma|+T_{\text {IQI,TDA-EDE }}\right)^{2}$ is greater than $|\gamma|^{2},\left(|\gamma|+T_{\text {IQI,TDA-EDE }}\right)^{2}$ is an upper error bound set by the validation for I/Q imbalance estimation. In low SNR region, CRLB exceeds this upper bound while NMSE of I/Q imbalance estimation keeps lower than it.

\section{Conclusions}

In this article, a novel blind estimator TDA-EDE for joint estimation of CFO, I/Q imbalance and DCO in DCA OFDM systems is presented. Compared with our previous study EDE, TDA-EDE reduces computation efforts to around $1 / 3$ of that of EDE by compensation of DCO with time domain average approach before estimation of CFO, and improves accuracy of I/Q imbalance estimation and DCO estimation by validation of estimated I/Q imbalance according to its practical range. Performance of TDA-EDE is demonstrated with established algorithms and CRLB by simulations.

\section{Acknowledgements}

This study was supported by the National Natural Science Foundation of China under Grant 60903004, the Beijing Natural Science Foundation under Grant 4102042 and the Fundamental Research Funds for the Central Universities under Grant FRF-TP-12-097A.

\section{Author details}

${ }^{1}$ School of Automation \& Electrical Engineering, University of Science and Technology Beijing, Beijing, 100083, China ${ }^{2}$ China Mobile Group Shandong Co., Ltd., Jinan, 250100, China

\section{Competing interests}

The authors declare that they have no competing interests.

Received: 20 January 2012 Accepted: 9 May 2012 Published: 9 May 2012

\section{References}

1. R Prasad, OFDM for Wireless Communications Systems, (Artech House, London, 2004)

2. H Steendam, M Moeneclaey, Sensitivity of orthogonal frequency-division multiplexed systems to carrier and clock synchronization errors. Signal Process. 80(7), 1217-1229 (2000). doi:10.1016/S0165-1684(00)00031-1

3. T Schmidl, D Cox, Robust frequency and timing synchronization for OFDM. IEEE Trans Commun. 45(12), 1613-1621 (1997). doi:10.1109/26.650240

4. J Van de Beek, M Sandell, P Borjesson, ML estimation of time and frequency offset in OFDM systems. IEEE Trans Signal Process. 45(7), 1800-1805 (1997). doi:10.1109/78.599949

5. H Liu, U Tureli, A high-efficiency carrier estimator for OFDM communications. IEEE Commun Lett. 2(4), 104-106 (1998)

6. U Tureli, H Liu, M Zoltowski, OFDM blind carrier offset estimation: ESPRIT. IEEE Trans Commun. 48(9), 1459-1461 (2000). doi:10.1109/26.870011

7. B Chen, Maximum likelihood estimation of OFDM carrier frequency offset. IEEE Signal Process Lett. 9(4), 123-126 (2002)

8. Y Yao, G Giannakis, Blind carrier frequency offset estimation in SISO, MIMO, and multiuser OFDM systems. IEEE Trans Commun. 53, 173-183 (2005). doi:10.1109/TCOMM.2004.840623

9. H Jeon, K Kim, E Serpedin, An efficient blind deterministic frequency offset estimator for OFDM systems. IEEE Trans Commun. 59(4), 1133-1141 (2011)

10. J Oh, J Kim, J Lim, Blind carrier frequency offset estimation for OFDM systems with constant modulus constellations. IEEE Commun Lett. 15(9), 971-973 (2011)

11. H Lin, HM Senevirathna, K Yamashita, Blind estimation of carrier frequency offset and DC offset for OFDM systems. IEEE Trans Commun. 56(5), 704-707 (2008)

12. T Liu, $H$ Li, Blind carrier frequency offset estimation in OFDM systems with I/Q imbalance. Signal Process. 89(11), 2286-2290 (2009). doi:10.1016/j. sigpro.2009.04.026

13. T Liu, H Li, Joint estimation of carrier frequency offset, dc offset and I/Q imbalance for OFDM systems. Signal Process. 91(5), 1329-1333 (2011). doi:10.1016/j.sigpro.2010.12.002

14. B Razavi, Design considerations for direct-conversion receivers. IEEE Trans Circ Syst II Analog Digital Signal Process. 44(6), 428-435 (1997). doi:10.1109/ 82.592569 
15. PI Mak, SP U, RP Martins, Transceiver architecture selection: review, state-ofthe-art survey and case study. IEEE Circ Syst Mag. 7(2), 6-25 (2007)

16. F Chastellain, C Botteron, P Farine, Looking inside modern receivers. IEEE Microwave Mag. 12(2), 87-98 (2011)

17. T Deepaknath, M Marc, Efficient compensation of transmitter and receiver IQ imbalance in OFDM systems. EURASIP J Adv Signal Process. 2010, 1-14 (2010)

18. J Tubbax, A Fort, L Van der Perre, S Donnay, M Engels, M Moonen, H De Man, Joint compensation of IQ imbalance and frequency offset in OFDM systems, in IEEE Global Telecommunications Conference (GLOBECOM), vol. 4. San Francisco, USA, pp. 2365-2369 (Dec 2003)

19. G Xing, M Shen, H Liu, Frequency offset and I/Q imbalance compensation for direct-conversion receivers. IEEE Trans Wirel Commun. 4(2), 673-680 (2005)

20. H Lin, X Wang, K Yamashita, A low-complexity carrier frequency offset estimator independent of DC offset. IEEE Commun Lett. 12(7), 520-522 (2008)

21. M Inamori, A Bostamam, Y Sanada, H Minami, IQ imbalance compensation scheme in the presence of frequency offset and dynamic DC offset for a direct conversion receiver. IEEE Trans Wirel Commun. 8, 2214-2220 (2009)

22. H Lin, X Zhu, K Yamashita, Low-complexity pilot-aided compensation for carrier frequency offset and I/Q imbalance. IEEE Trans Commun. 58(2), 448-452 (2010)

23. Y Chung, S Phoong, Joint estimation of I/Q imbalance, CFO and channel response for MIMO OFDM systems. IEEE Trans Commun. 58(5), 1485-1492 (2010)

24. F Yan, WP Zhu, MO Ahmad, Carrier frequency offset estimation and I/Q imbalance compensation for OFDM systems. EURASIP J Adv Signal Process. 2007, 1-11 (2007)

25. GH Golub, CFV Loan, Matrix Computations, (JHU Press, Baltimore, 1996)

26. S Smale, Complexity theory and numerical analysis. Acta Numerica. 6, 523-551 (1997)

27. J Demmel, I Dumitriu, O Holtz, Fast linear algebra is stable. Numerische Mathematik. 108, 59-91 (2007). doi:10.1007/s00211-007-0114-x

28. Part 11, Wireless LAN medium access control and physical layer (PHY) specifications: high-speed physical layer in the $5 \mathrm{GHz}$ band. IEEE 802 LAN/ MAN Standards (1999)

doi:10.1186/1687-6180-2012-105

Cite this article as: Liu and Li: Blind estimation of carrier frequency

offset, I/Q imbalance and DC offset for OFDM systems. EURASIP Journal

on Advances in Signal Processing 2012 2012:105.

\section{Submit your manuscript to a SpringerOpen ${ }^{\circ}$ journal and benefit from:}

- Convenient online submission

- Rigorous peer review

- Immediate publication on acceptance

- Open access: articles freely available online

- High visibility within the field

- Retaining the copyright to your article

Submit your next manuscript at $\gg$ springeropen.com 